\title{
Identification of guanine nucleotide-binding protein $\gamma-7$ as an epigenetically silenced gene in head and neck cancer by gene expression profiling
}

\author{
SEMRA DEMOKAN ${ }^{1,5}$, ALICE Y. CHUANG ${ }^{2}$, XIAOFEI CHANG ${ }^{1}$, TANBIR KHAN $^{1}$, IAN M. SMITH ${ }^{1}$, \\ KAVITA M. PATTANI ${ }^{1}$, SANTANU DASGUPTA ${ }^{1,6}$, SHAHNAZ BEGUM ${ }^{3}$, ZUBAIR KHAN ${ }^{1}$, \\ NANETTE J. LIEGEOIS ${ }^{2}$, WILLIAM H. WESTRA ${ }^{3}$, DAVID SIDRANSKY ${ }^{1}$, \\ WAYNE $\mathrm{KOCH}^{1}$ and JOSEPH A. CALIFANO ${ }^{1,4}$
}

\begin{abstract}
Departments of ${ }^{1}$ Otolaryngology-Head and Neck Surgery, ${ }^{2}$ Dermatology and ${ }^{3}$ Pathology, Johns Hopkins Medical Institutions, Baltimore, MD; ${ }^{4}$ Milton J. Dance Head and Neck Center, Greater Baltimore Medical Center, Baltimore, MD, USA; ${ }^{5}$ Department of Basic Oncology, Oncology Institute, Istanbul University, Capa, Istanbul, Turkey; ${ }^{6}$ Department of Human and Molecular Genetics, Virginia Commonwealth University, Richmond, VA, USA
\end{abstract}

Received November 1, 2012; Accepted December 10, 2012

DOI: 10.3892/ijo.2013.1808

\begin{abstract}
Silencing of tumor suppressor genes plays a vital role in head and neck carcinogenesis. Aberrant hypermethylation in the promoter region of some known or putative tumor suppressor genes occurs frequently during the development of various types of cancer including head and neck squamous cell carcinoma (HNSCC). In this study we used an expanded mRNA expression profiling approach followed by microarray expression analysis to identify epigenetically inactivated genes in HNSCC. Two HNSCC cell lines were treated with 5-aza2'-deoxycytidine followed by microarray analysis to identify epigenetically silenced genes in HNSCC. We found 1,960, 614 and 427 genes were upregulated in the HNSCC cell lines JHU-012, JHU-011 and the combination of both cell lines, respectively. HNSCC tumor and normal mucosal samples were used for gene profiling by a $47 \mathrm{~K}$ mRNA gene expression array and we found 7,140 genes were downregulated in HNSCC tumors compared to normal mucosa, as determined by microarray analysis, and were integrated with cell line data. Integrative analysis defined 126 candidate genes, of which only seven genes showed differential methylation in tumors and no methylation in normal mucosa after bisulfite sequencing. Following validation by QMSP, one gene, guanine nucleotide-binding protein $\gamma-7$ (GNG7), was confirmed to be highly methylated in tumors and unmethylated in normal mucosal and salivary rinse samples demonstrating cancer-
\end{abstract}

Correspondence to: Dr Semra Demokan, Department of Basic Oncology, Oncology Institute, Istanbul University, Capa, 34093 Istanbul, Turkey

E-mail: demokan@istanbul.edu.tr

Key words: guanine nucleotide-binding protein $\gamma-7$, gene expression, silencing, head and neck squamous cell carcinoma, epigenetics specific methylation in HNSCC tissues. TXNIP and TUSC2 were partially methylated in tumors and normal salivary rinses but unmethylated in normal mucosa. We concluded that GNG7 is a highly specific promoter methylated gene associated with HNSCC. In addition, TXNIP and TUSC 2 are also potential biomarkers for HNSCC.

\section{Introduction}

Among human malignancies, head and neck cancer is the sixth most common cancer in the world (1). Head and neck cancer is an aggressive and life-threatening disease with poor morbidity and high mortality in advanced disease. More than 40,000 new cases of head and neck squamous cell carcinoma (HNSCC) are diagnosed in the United States each year, with 12,000 US deaths annually. Survival rates have not improved significantly for patients with HNSCC in the past thirty years despite active clinical and basic research addressing this issue. Treatment for HNSCC includes surgical resection, chemotherapy and radiation therapy; however, approximately $50 \%$ of all patients have advanced disease at the time of diagnosis often requiring use of all three treatment modalities. Therefore, it is important to discover new biomarkers in a cancer-specific manner and to develop new methods that provide sensitive and reliable biomarkers of HNSCC for detection, treatment response and prognosis.

Genetic alterations are a hallmark of human cancer, with the activation of proto-oncogenes and inactivation of tumor suppressor genes, either through deletion or inactivating point mutations, being well defined (2). In addition to these genetic alterations, changes in DNA methylation, an epigenetic process present in mammalian cells, are also a hallmark of human cancer (3). Silencing of tumor suppressor genes by means of promoter hypermethylation plays an important role in head and neck carcinogenesis (4). Methylation of the $\mathrm{CpG}$ islands in the promoter regions of tumor suppressor genes is frequently observed with resultant reduced gene expression $(5,6)$. 
To discover the new cancer-specific hypermethylated genes, gene expression profiling via oligonucleotide microarray-based approach is a reliable technology for whole genome epigenetic research (7). Measuring promoter hypermethylation by using real-time quantitative methylation-specific PCR (QMSP) allows an objective, robust, and rapid assessment of promoter methylation status (8-11).

Previously, we employed a pharmacologic unmasking expression array technique using a $12 \mathrm{~K}$ gene expression array to identify epigenetically inactivated genes in HNSCC (12). In this study, we expanded this approach using a whole genome $47 \mathrm{~K}$ array platform and then performed bisulfite DNA sequencing for 126 selected genes and QMSP for seven selected genes after evaluating the bisulfite sequencing results, to validate HNSCC-specific methylation in novel genes.

\section{Materials and methods}

Cell lines. We used 2 human head and neck cancer cell lines, JHU-011 and JHU-012, which were developed from a laryngeal primary and a neck node metastasis of different HNSCC patients, at the Department of Otolaryngology-Head and Neck Surgery, Johns Hopkins University. Cell lines were cultured in RPMI-1640 medium supplemented with $10 \%$ fetal bovine serum and $1 \%$ penicillin-streptomycin. All media components were obtained from Life Technologies Invitrogen Corp. All cell lines tested negative for any mycoplasma contamination.

5-aza-2'-deoxycytidine treatment. Cell lines were treated with 5-aza-2'-deoxycytidine (5-aza-dC, a demethylating agent) and trichostatin A (TSA, a histone deacetylase inhibitor) as previously described $(7,12)$. Briefly, we seeded all cell lines $\left(1 \times 10^{6}\right)$ in their respective culture medium and maintained them for $24 \mathrm{~h}$ before treating them with $5 \mu \mathrm{M} 5$-aza-dC (Sigma, St. Louis, MO, USA) for 5 days and $300 \mathrm{nM}$ for the final $24 \mathrm{~h}$. We renewed medium containing 5 -aza-dC every $24 \mathrm{~h}$ during the treatment and handled control cells similarly, without adding 5-aza-dC. Stock solutions of 5-aza-dC (Sigma) and TSA (Sigma) were dissolved in DMSO (Sigma) and ethanol (100\%), respectively.

Tissue samples. After obtaining institutional review board approval and appropriate informed consent, the HNSCC patients and control population (healthy subjects enrolled in a community screening study) were recruited at the Johns Hopkins School of Medicine, Department of OtolaryngologyHead and Neck Surgery. Five normal mucosa samples from healthy individuals by uvulopalatopharyngoplasty (UPPP) technique, 13 HNSCC tumors for mRNA expression array experiments, 22 salivary rinses and 14 mucosal samples from a healthy population and 33 HNSCC tumor samples were collected. Salivary rinses were obtained by brushing oral cavity and oropharyngeal surfaces with an exfoliating brush followed by rinse and gargle with $20 \mathrm{ml}$ normal saline solution. The brush was gently agitated to release the obtained material into saline. Following centrifugation, the supernatant was discarded and DNA was isolated from the pellet. Tumors were snap frozen and microdissected on a cryostat to $\geq 75 \%$ purity. DNA from 22 salivary rinse samples and 14 normal mucosa samples from healthy individuals were analyzed as a control, to investigate the normal promoter methylation status of seven newly identified candidate genes, $M A P 2 K 3$ (mitogen-activated protein kinase kinase 3) $(\mathrm{n}=18), M A P 3 K 3$ (mitogen-activated protein kinase kinase kinase 3) $(\mathrm{n}=18)$, GNG7 (guanine nucleotide-binding protein, $\gamma-7)(\mathrm{n}=17)$, GALNT10 (UDP-N-acetyl- $\alpha$-D-galactosamine:polypeptide $\mathrm{N}$-acetylgalactosaminyltransferase 10$)(\mathrm{n}=18)$, PPFIBP2 (PTPRF interacting protein, binding protein 2) $(\mathrm{n}=17)$, TUSC2 (tumor suppressor candidate 2) $(\mathrm{n}=16)$ and TXNIP (thioredoxin interacting protein) $(\mathrm{n}=16)$. The methylation status of these genes was analyzed in 33 fresh HNSCC tumor samples.

RNA isolation, cDNA synthesis and probe hybridization. We prepared total RNA from 13 HNSCC tumors, 5 normal mucosa samples and cell lines using the RNeasy Mini kit (Qiagen, Valencia, CA, USA). Total RNA quality was checked via the Nanodrop Spectrophotometer and Agilent Bioanalyzer total RNA series II kit. Total RNA ( $1 \mu \mathrm{g})$ was combined with $2 \mu 1 \mathrm{~T} 7$ oligo(dT) primer and $2 \mu \mathrm{l}$ Poly-A controls and brought to a volume of $12 \mu \mathrm{l}$. The samples were incubated at $70^{\circ} \mathrm{C}$ for 10 min. A master mix of $4 \mu 1$ first strand buffer, $2 \mu 1$ DTT and $1 \mu 110 \mathrm{mM}$ dNTPs was added to the samples, followed by a 2 -min incubation at $42^{\circ} \mathrm{C}$. Superscript II (Invitrogen, Carlsbad, CA, USA) $(1 \mu \mathrm{l})$ was added to each sample and the samples were incubated at $42^{\circ} \mathrm{C}$ for $1 \mathrm{~h}$ for first strand cDNA synthesis. A master mix of $91 \mu 1$ water, $30 \mu 15 \mathrm{X}$ second strand buffer, $3 \mu \mathrm{l} 10 \mathrm{mM}$ dNTPs, $4 \mu \mathrm{l}$ DNA polymerase I, $1 \mu \mathrm{l} E$. coli DNA ligase and $1 \mu 1 \mathrm{RNase} \mathrm{H}$ was added to each sample and the samples were incubated for $2 \mathrm{~h}$ at $16^{\circ} \mathrm{C}$ for second strand cDNA synthesis. T4 DNA polymerase $(2 \mu 1)$ was added followed by a 5 -min incubation at $16^{\circ} \mathrm{C}$. EDTA $(10 \mu 1,0.5 \mathrm{M})$ was added to stop the reaction. cDNA cleanup was performed with the Affymetrix GeneChip Sample Cleanup Module, according to the manufacturer's instructions. The cDNA was eluted in $14 \mu \mathrm{l}$ elution buffer. The final elution volume $(\sim 12 \mu \mathrm{l})$ was combined with $28 \mu \mathrm{l}$ of IVT mix master mix (4 $\mu 1$ 10X IVT labeling buffer, $12 \mu 1$ labeling NTP mix, $4 \mu 1$ labeling enzyme mix, $8 \mu 1$ RNAse-free water) for the in vitro transcription cRNA synthesis reaction. The samples were incubated overnight for $16 \mathrm{~h}$ at $37^{\circ} \mathrm{C}$ followed by a hold at $4^{\circ} \mathrm{C}$. Cleanup was performed as per the manufacturer's protocol using the Affymetrix GeneChip Sample Cleanup Module. The final elution volume was $\sim 19 \mu \mathrm{l}$. Concentration was checked via a nanodrop spectrophotometer. Fragmentation buffer (5X) (6 $\mu \mathrm{l})$ was then combined with $15 \mu \mathrm{g}$ cRNA and incubated at $95^{\circ} \mathrm{C}$ for $35 \mathrm{~min}$ to fragment the cRNA. The samples were ice quenched and combined with the hybridization cocktail. After 10 min of pre-hybridizing Human U133 Plus 2.0 Genome array at $45^{\circ} \mathrm{C}, 60 \mathrm{rpm}, 200 \mu \mathrm{l}$ of cocktail was loaded onto each array and the arrays were hybridized for $16 \mathrm{~h}$ at $45^{\circ} \mathrm{C}, 60 \mathrm{rpm}$. The cocktail was removed and the arrays were stained and washed using the Affymetrix GeneChip Fluidics Station 450 and FS450_001 fluidics script. All arrays were scanned in the Affymetrix GeneChip Scanner 3000 and the raw analysis was performed with Affymetrix GeneChip Operating System (GCOS) 1.4. Following RNA isolation, the expertise, facilities and instrumentation for Affymetrix GeneChip experimentation were performed at the JHU microarray core facility. All reagents needed for CDNA synthesis and probe hybridization were provided by Affymetrix (Santa Clara, CA, USA) 
Microarray data analysis. mRNA gene expression profiling was performed using Affymetrix GeneChip Human Genome U133 Plus 2.0 Arrays containing 47K probe sets (Affymetrix). Signal intensity and statistical significance were established for each transcript initially using dChip version 2008 (13) and then significance analysis of microarrays (SAM) (14) software to analyze and normalize the array data. Default settings for dChip were used, including the perfect match/mismatch difference model, invariant set normalization and check single/probe/array outlier algorithm. The mRNA gene expression profiling data has been deposited in the Gene Expression Omnibus (GEO) database with the accession no. GSE29330.

DNA extraction and bisulfite treatment. DNA was isolated as previously described (15). In brief, DNA was obtained by phenol/chloroform extraction following overnight incubation with proteinase $\mathrm{K}$ (Boehringer-Mannheim, Germany) at $48^{\circ} \mathrm{C}$. DNA from tumor and control samples was subjected to bisulfite treatment using Epitect Bisulfite Modification kit (Qiagen) according to the manufacturer's instructions.

Bisulfite sequencing. Bisulfite sequence analysis was performed to determine the methylation status in the promoter regions of 126 genes obtained from gene expression profiling in normal mucosal and HNSCC tumor samples. Bisulfite-treated DNA was amplified for the $5^{\prime}$ region that included at least a portion of the $\mathrm{CpG}$ island within $1-2 \mathrm{~kb}$ of the first exon of 126 genes, using primer sets (data not shown). The promoter regions of the genes were found from the database of the University of California, Santa Cruz, USA (UCSC) (http://genome.ucsc. $\mathrm{edu} /$ ). Primer sequences were determined by the MethPrimer program (16) showing the $\mathrm{CpG}$ islands in the promoter regions of 126 genes for bisulfite sequencing. The primers for bisulfite sequencing were designed to hybridize to regions in the promoter without $\mathrm{CpG}$ dinucleotides. PCR products were gel-purified using the QIAquick Gel Extraction kit (Qiagen) according to the manufacturer's instructions. Each amplified DNA sample was sequenced by the Applied Biosystems 3700 DNA analyzer using nested, forward or reverse primers and BD terminator dye (Applied Biosystems, Foster City, CA, USA).

Quantitative methylation-specific PCR. To determine if the methylated genes in tumor samples were cancer-specific, we investigated promoter methylation in 22 normal saliva, 14 agematched normal mucosa from healthy individuals that were analyzed as a control, to investigate the normal promoter methylation status of seven newly identified candidate genes (MAP3K3, MAP2K3, GNG7, GALNT10, PPFIBP2, TUSC2 and $T X N I P$ ) and in 33 HNSCC tumor samples by QMSP. Primer and probe sequences were determined by the MethPrimer program showing the $\mathrm{CpG}$ islands in the promoter regions of seven genes selected after bisulfite sequencing (Table I). Lymphocytes obtained from a healthy individual were in vitro methylated using excess SssI methyltransferase (New England Biolabs Inc., Beverly, MA, USA) to generate completely methylated DNA that was used as a positive control standard. To quantitate the relative percent of methylation, we computed the ratio between the QMSP values of the gene of interest relative to an internal control, ACTB (15) ( $\beta$-actin) (Table I) (gene of interest/reference gene x 100). Fluorogenic PCR was carried

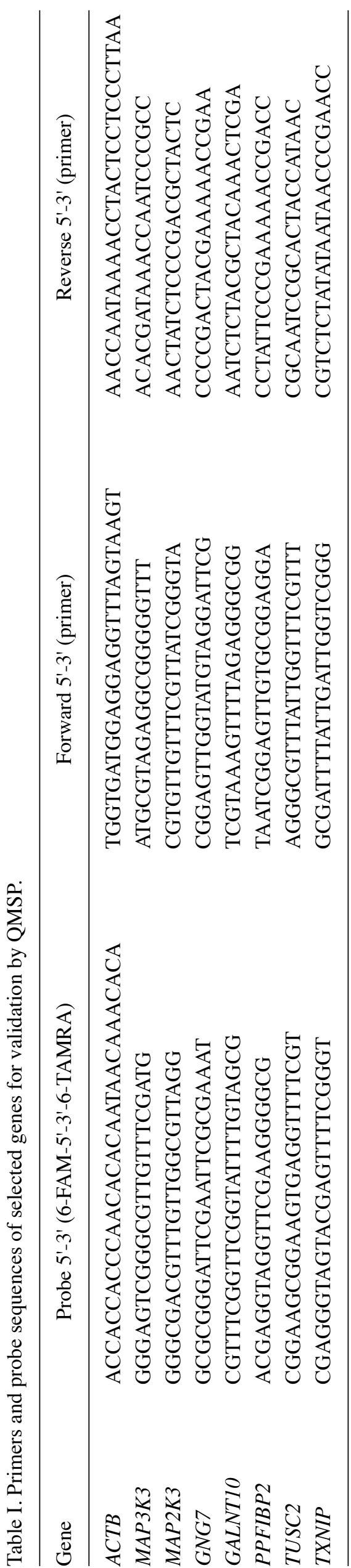




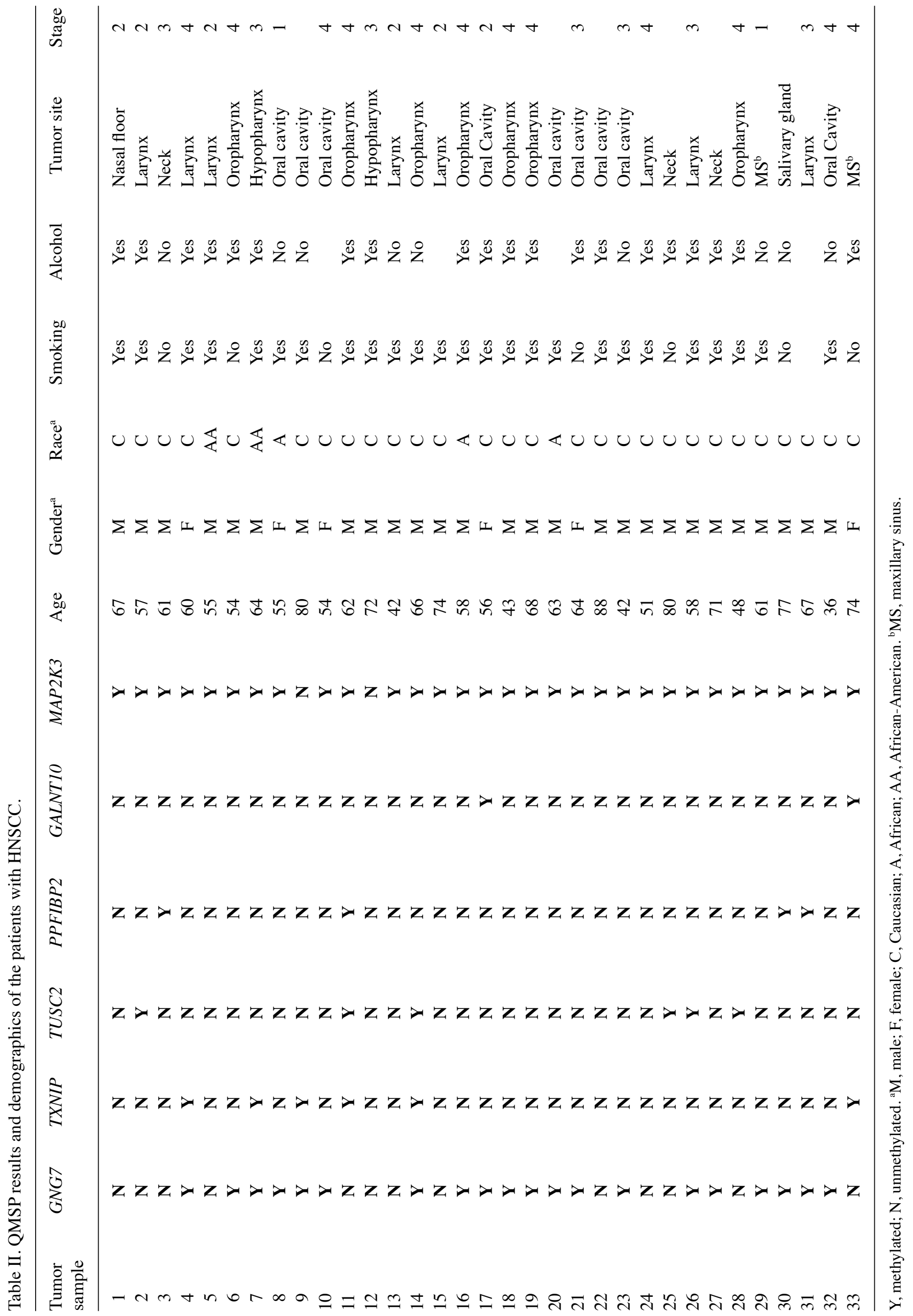


Table III. Clinical characteristics of mRNA expression array cohort.

\begin{tabular}{cccccccccccc}
\hline Case & Diagnosis & Age & Gender $^{\mathrm{a}}$ & Race $^{\mathrm{a}}$ & Overall stage & $\mathrm{T}$ & $\mathrm{N}$ & $\mathrm{M}$ & Site & Tobacco & Alcohol \\
\hline 1 & Normal & 20 & $\mathrm{M}$ & $\mathrm{C}$ & NA & NA & NA & NA & Left tonsil & No & No \\
2 & Normal & 28 & $\mathrm{M}$ & $\mathrm{C}$ & NA & NA & NA & NA & Right tonsil & No & No \\
3 & Normal & 28 & $\mathrm{M}$ & $\mathrm{C}$ & NA & NA & NA & NA & Uvula & No & No \\
4 & Normal & 28 & M & C & NA & NA & NA & NA & Left tonsil & NA & NA \\
5 & Normal & 30 & M & C & NA & NA & NA & NA & Uvula & No & No \\
6 & Cancer & 62 & M & C & 3 & 3 & 0 & 0 & Larynx & No & Yes \\
7 & Cancer & 80 & F & C & 1 & 1 & $2 A$ & 0 & Oral cavity & No & NA \\
8 & Cancer & NA & NA & NA & NA & NA & NA & NA & NA & NA & NA \\
9 & Cancer & 82 & M & C & 3 & 3 & 0 & 0 & Larynx & Yes & NA \\
10 & Cancer & 74 & M & C & NA & NA & NA & NA & NA & NA & NA \\
11 & Cancer & 71 & M & C & 4 & 4 & 0 & 0 & Oral cavity & Yes & No \\
12 & Cancer & 56 & M & AA & NA & NA & NA & NA & NA & NA & NA \\
13 & Cancer & 62 & F & AA & 1 & 1 & 0 & 0 & Oral cavity & Yes & Yes \\
14 & Cancer & 58 & M & C & 3 & 2 & $2 B$ & 0 & Oropharynx & No & Yes \\
15 & Cancer & 61 & M & C & NA & NA & NA & NA & NA & NA & NA \\
16 & Cancer & 89 & M & C & NA & NA & NA & NA & NA & Yes & Yes \\
17 & Cancer & 63 & M & C & 2 & 2 & 0 & 0 & Larynx & Yes & Yes \\
18 & Cancer & 50 & F & C & 4 & 3 & $2 B$ & 0 & Oropharynx & Yes & Yes \\
\hline
\end{tabular}

${ }^{\mathrm{a}} \mathrm{M}$, male; F, female; C, Caucasian; A, African; AA, African-American; NA, not available. T, tumor; N, node; M, metastasis.

out in a reaction volume of $20 \mu \mathrm{l}$ consisting of $600 \mathrm{nM}$ of each primer; $200 \mathrm{nM}$ of probe; $0.6 \mathrm{U}$ of platinum Taq polymerase (Invitrogen); $200 \mu \mathrm{M}$ of each dATP, dCTP, dGTP and dTTP; $1 \mathrm{X}$ Rox Dye reference and $1 \mathrm{X}$ buffer [16.6 $\mathrm{mM}$ of ammonium sulfate; $67 \mathrm{mM}$ of Trizma (Sigma); $6.7 \mathrm{mM}$ of magnesium chloride; $10 \mathrm{mM}$ of mercaptoethanol; and $0.1 \%$ dimethylsulfoxide]. Thirty nanograms of bisulfite-treated DNA were used in each real-time QMSP reaction. Amplifications were carried out in 384-well plates in a 7900 Sequence Detector system (PerkinElmer Applied Biosystems, Norwalk, CT, USA) and were analyzed by SDS 2.3 (Sequence Detector System) (Applied Biosystems). Each reaction was performed in triplicate.

\section{Results}

Clinicopathological characteristics of control subjects and patients with HNSCC. Table II describes the demographic parameters of the sample populations used in this study. The mean age of normal mucosal subjects was 43.4 years (range 24-65). Forty-two percent of controls were tobacco users. Normal mucosal and tumor subjects had a similar male and Caucasian predominance. Among tumor patients, smoking rate was $78 \%$ and alcohol consumption was $69 \%$. Tumor samples $(\mathrm{n}=33)$ were obtained from patients with stage I $(7.4 \%)$, stage II (22\%), stage III (26\%) and stage IV (44\%) lesions. These were from primary tumors of the oral cavity $(n=9)$, oropharynx $(n=7)$, hypopharynx $(n=2)$, larynx $(n=8)$, maxillary sinus $(n=2)$, nasal floor $(n=1)$, salivary gland $(n=1)$ and unknown primary/ neck $(n=3)$. Male and Caucasian status was less prevalent in the normal salivary rinse subjects and $36 \%$ were tobacco users. Individuals from whom the normal salivary rinse was obtained were slightly younger than the population of head and neck cancer patients, with a mean age of 52.2 years (range 19-83) and 61.4 years (range 36-88), respectively. In Table III, the clinical findings of the mRNA expression cohort are given.

Workflow of gene methylation discovery approach. We performed pharmacological unmasking analysis on two HNSCC cancer cell lines JHU-O11 and JHU-O12 by treating cells with or without 5-aza-dC (as a control group), followed by RNA extraction and microarray analysis using Affymetrix U133 Plus 2.0. The array data were analyzed initially by dChip and then SAM. We performed a four-phase strategy to obtain the unmasked genes in the cells treated with 5-aza and downregulated genes in primary tumors. In the first phase, we compared the cell lines, either JHU-012 or JHU-011, before treatment to the cell lines treated with 5 -aza, in order to identify genes that were reexpressed $\geq 2$-fold. We found 1,960 genes that were upregulated by 5 -aza-dC in the JHU-O12 cell line. SAM output was obtained at a delta value of 2.05 with a false discovery rate (FDR) of $10 \%$ and the d-score cut-off was 1.17 . We found 614 reexpressed genes in 5-aza-treated JHU-O11 (SAM output; delta=2.089, FDR=10\%, d-score cut-off=2.8); 427 genes were commonly upregulated in both cell lines when the cell lines were normalized and analyzed together (SAM output; delta=1.44, FDR=10\%, d-score cut-off=1.88) (Fig. 1). In the second phase of our analysis, we further extracted RNA and performed the $47 \mathrm{~K}$ mRNA expression array analysis on 13 primary HNSCC tumors and 5 normal mucosal samples from non-cancer control patients. Following initial dChip 
Table IV. The results of SAM analysis in human and cell line specimens.

Downregulated genes from the SAM output of human tumor vs. normal mucosal tissues
Upregulated genes from the SAM analysis of 5-aza non-treated vs. 5-aza-treated JHU-011 + JHU-012 cells

\begin{tabular}{llccccccccc}
\hline Rank & Gene ID & Score $(\mathbf{d})$ & Fold change & q-value $(\boldsymbol{\%})$ & Rank & Gene ID & Score (d) & Fold change & q-value $(\boldsymbol{\%})$ \\
\hline 5506 & GALNT10 & $\mathbf{- 2 . 8 4}$ & $\mathbf{0 . 5 5}$ & 3.37 & 61 & $G A L N T 10$ & $\mathbf{8 . 3 8}$ & $\mathbf{2 . 2 8}$ & 2.55 \\
5189 & $M A P 2 K 3$ & $\mathbf{- 2 . 9 2}$ & $\mathbf{0 . 6 5}$ & 2.89 & 13 & $M A P 2 K 3$ & $\mathbf{1 2 . 0 7}$ & $\mathbf{2 . 5 5}$ & 0.00 \\
3406 & $M A P 3 K 3$ & $\mathbf{- 3 . 4 4}$ & $\mathbf{0 . 7 3}$ & 1.47 & 309 & $M A P 3 K 3$ & $\mathbf{5 . 1 5}$ & $\mathbf{2 . 1 2}$ & 7.68 \\
1939 & PPFIBP2 & $\mathbf{- 3 . 9 9}$ & $\mathbf{0 . 5 8}$ & 0.86 & 389 & $P P F I B P 2$ & $\mathbf{4 . 7 5}$ & $\mathbf{1 . 9 9}$
\end{tabular}

Upregulated genes from the SAM analysis of non-treated vs. 5-aza-treated JHU-012 cell lines

\begin{tabular}{|c|c|c|c|c|c|c|c|c|c|}
\hline 34 & GNG7 & -8.32 & 0.21 & 0.00 & 313 & GNG7 & 13.95 & 5.34 & 4.02 \\
\hline 813 & TUSC2 & -4.82 & 0.57 & 0.37 & 408 & TUSC2 & 12.02 & 1.44 & 4.56 \\
\hline & & & & & \multicolumn{5}{|c|}{$\begin{array}{l}\text { Upregulated gene from the SAM analysis of } \\
\text { non-treated vs. 5-aza-treated JHU-011 cell lines }\end{array}$} \\
\hline 317 & TXNIP & -4.32 & 0.36 & 0.61 & 362 & $T X N I P$ & 9.55 & 5.39 & 7.40 \\
\hline
\end{tabular}

Discovery Strategy (mRNA expression arrays,12K and 47K, Affymetrix U133Plus 2.0)

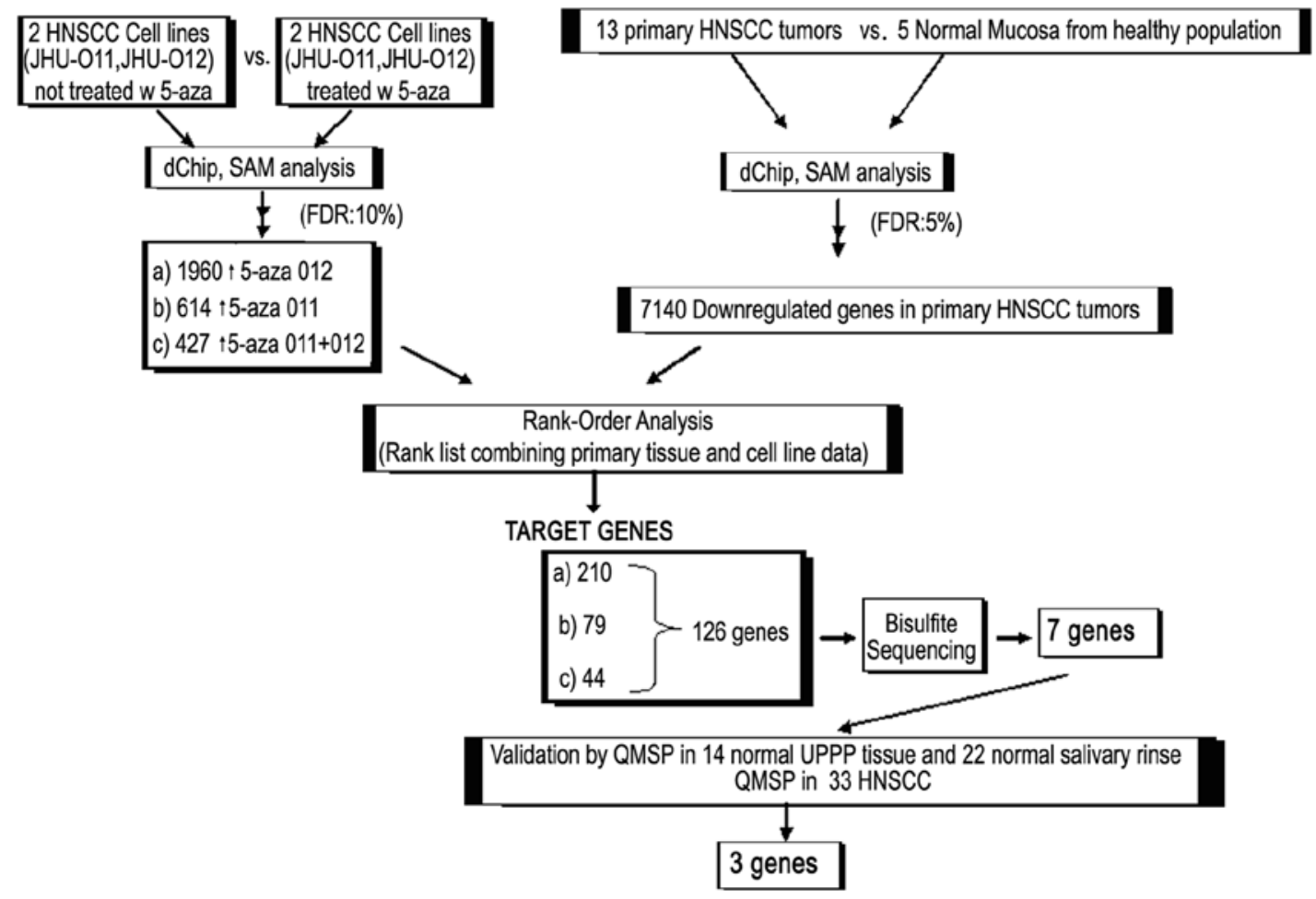

Figure 1. The workflow of discovery approach via gene expression array technology.

and SAM analysis (SAM output; delta=1.247, FDR $=10 \%$, d-score cut-off=0.24), we found 7,140 downregulated genes in primary HNSCC tumors compared with normal mucosa. In the third phase, we investigated the three data sets (Fig. 1) below: a) SAM output of 1,960 upregulated genes after 5-aza treatment of JHU-012 vs. SAM output of 7,140 downregulated genes in primary HNSCC. We found that 210 genes that were upregulated by 5 -aza-dC in the JHU-O12 cell line and showed downregulation in tumor samples. b) SAM output of 614 upregulated genes after 5-aza treatment of JHU-011 vs. SAM output of 7,140 downregulated genes in primary HNSCC. We found 79 genes that were upregulated by 5 -aza-dC in the JHU-O11 cell line and showed downregulation in tumor samples. c) SAM output from analyzing both cell lines together in the same SAM computation, of 427 upregulated genes after 5-aza treatment of JHU-011 and JHU-012 vs. SAM output of 
A

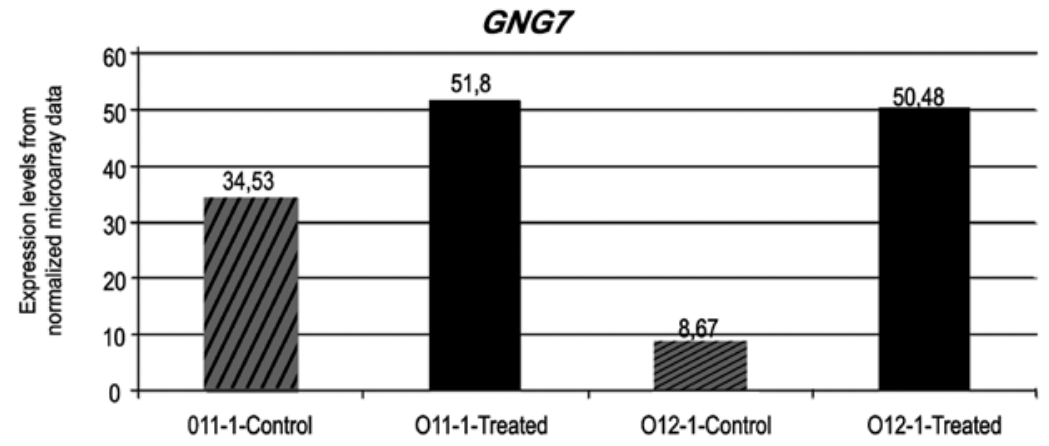

B

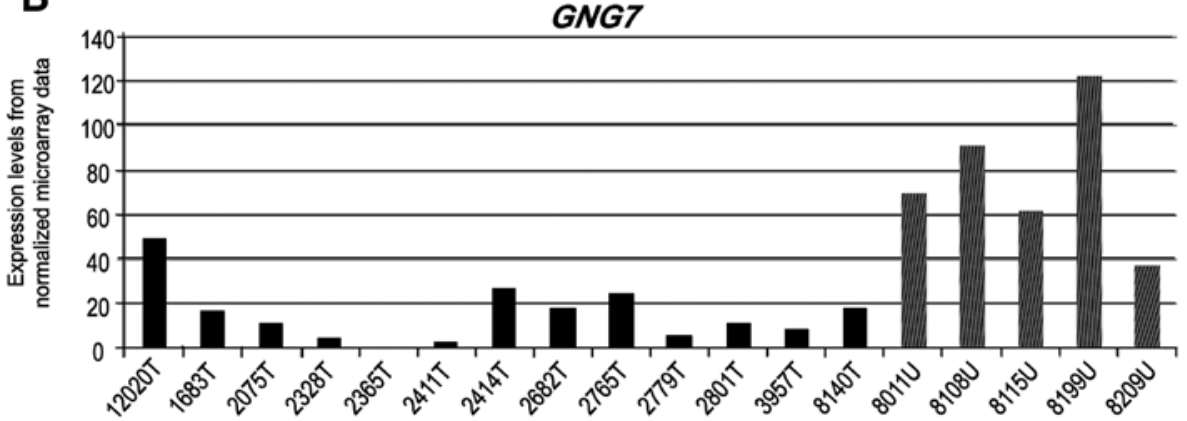

Figure 2. Expression array values from normalized microarray results of the GNG7 gene: (A) from cell lines treated with 5-aza and non-treated with 5-aza; (B) human tumors and normal tissue samples.

A
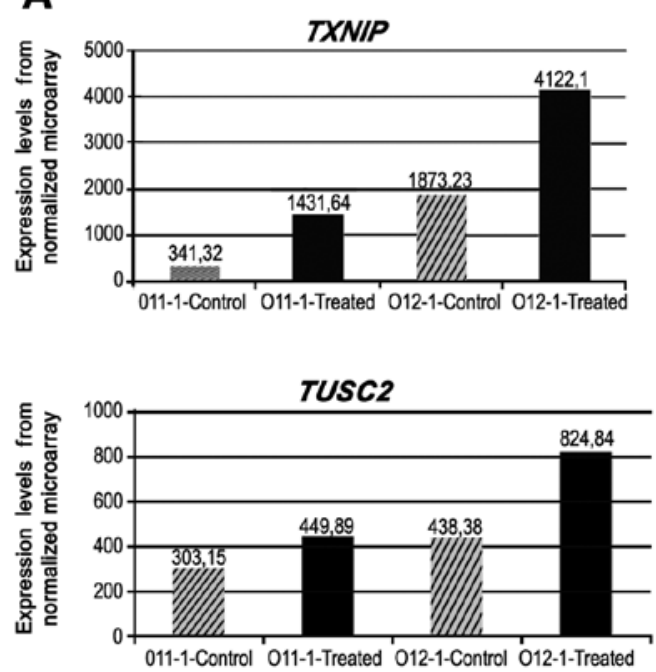

B
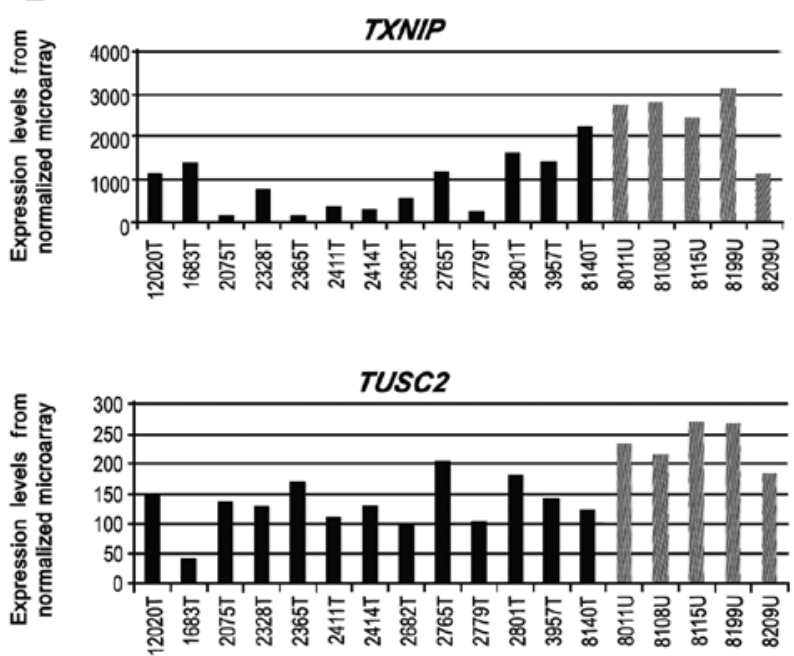

Figure 3. Expression array values from normalized microarray results of the TXNIP and TUSC2 genes: (A) from cell lines treated with 5-aza and non-treated with 5-aza; (B) human tumors and normal tissue samples.

7,140 downregulated genes in primary HNSCC. We found 44 genes that were upregulated by 5 -aza-dC in the JHU-O11 and JHU-012 cell lines and showed downregulation in tumor samples, suggesting that methylation might be involved in gene downregulation.

In the fourth phase of our strategy, we rank-ordered the results of upregulated genes obtained from these 3 data sets and found 126 common genes. We then examined promoter regions of the 126 genes for $\mathrm{CpG}$ islands and performed bisulfite sequencing analysis of the promoter region of these genes. We found that seven genes showed a differential meth- ylation pattern between normal and neoplastic samples (Fig. 1). Table IV shows the d-scores, fold change and q-values of these genes by SAM analysis in human and cell line samples. After validation of these genes in a cohort of $33 \mathrm{HNSCC}$ patients and normal salivary and mucosal samples from healthy individuals by QMSP, we found 3 genes of interest (GNG7, TXNIP and TUSC2). The upfold expression arrays of these genes are given in Figs. 2 and 3. A 1.5-fold upregulation in GNG7 expression was observed in 5-aza-treated JHU-011, whereas the rate of change was 5.8-fold in 5-aza-treated metastatic JHU-012 cells (Fig. 2). The TXNIP gene was upregulated 4.19-fold in JHU-011 
Table V. Methylation analysis of candidate genes in 4 normal mucosa and 4 tumor samples by bisulfite sequencing.

\begin{tabular}{|c|c|c|c|c|c|}
\hline Probe name & Gene ref ID & Gene name & Chromosome location & $\begin{array}{l}\text { Normal mucosa } \\
\text { tissue, } \mathrm{n}(\%)\end{array}$ & $\begin{array}{l}\text { HNSCC tumor } \\
\text { tissue, n }(\%)\end{array}$ \\
\hline 215499_at & NM_145109 & $M A P 2 K 3$ & $\operatorname{chr} 17: 21,128,561-21,159,144$ & 0 of $4(0)$ & 4 of $4(100)$ \\
\hline 203514_at & NM_002401 & $M A P 3 K 3$ & chr17:59,053,533-59,127,402 & 0 of $4(0)$ & 4 of $4(100)$ \\
\hline 220296_at & NM_198321 & GALNT10 & chr5:153,550,488-153,780,003 & 0 of $4(0)$ & 4 of $4(100)$ \\
\hline 201010_s_at & NM_006472 & $T X N I P$ & chr1:144,149,819-144,153,985 & 0 of $4(0)$ & 4 of $4(100)$ \\
\hline 203273_s_at & NM_007275 & TUSC2 & chr3:50,337,345-50,340,672 & 0 of $4(0)$ & 4 of $4(100)$ \\
\hline 212841_s_at & NM_003621 & $P P F I B P 2$ & chr11:7,491,577-7,631,567 & 0 of $4(0)$ & 2 of $4 \quad(50)$ \\
\hline 206896_s_at & NM_052847 & GNG7 & chr19:2,462,218-2,653,746 & 0 of $4(0)$ & 1 of 3 \\
\hline
\end{tabular}
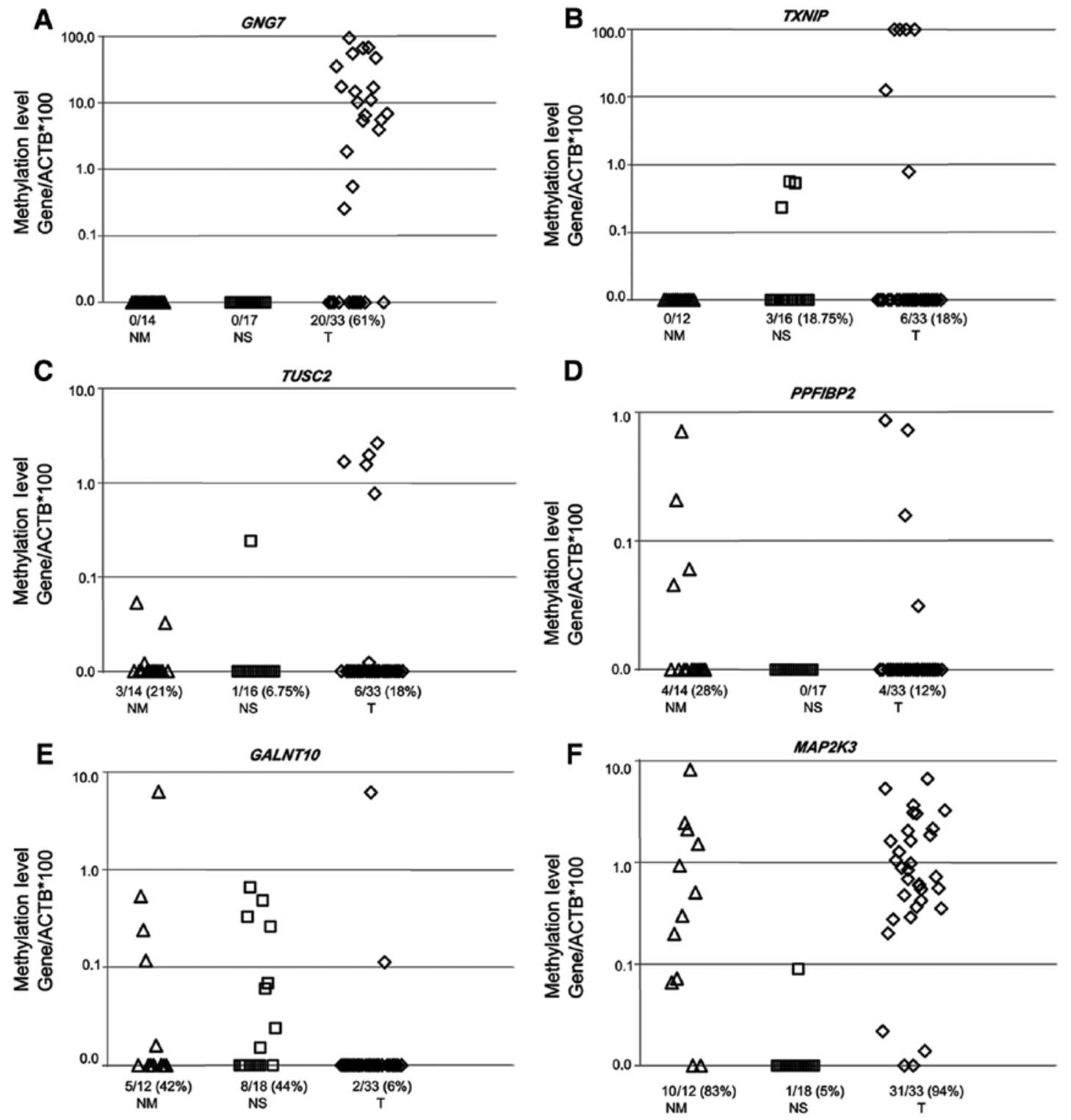

Figure 4. Scatter graph displaying the distribution of the methylation levels of 6 candidate genes: (A) GNG7; (B) TXNIP; (C) TUSC2; (D) PPFIBP2; (E) GALNT10; (F) MAP2K3 in normal mucosa samples (NM), normal salivary rinses (NS) and HNSCC tumors (T).

cells, whereas a 2.2-fold change was observed in the JHU-012 cells. The TUSC2 gene was upregulated in a similar manner in both cell lines (Fig. 3).
Genes specifically methylated in HNSCC tumors. We then performed bisulfite sequencing analysis on the promoter region of 126 genes as described above in our discovery 
approach using 4 normal mucosal and 4 HNSCC samples. Seven genes (GNG7, GALNT10, TXNIP, TUSC2, PPFIBP2, $M A P 2 K 3$ and $M A P 3 K 3$ ) were found to have no methylation in normal mucosal samples but displayed high methylation frequency in HNSCC samples (Table V). This high specificity prompted us to further investigate the methylation frequency in a larger cohort of normal mucosal and HNSCC specimens. We then performed QMSP on 22 saliva and 14 normal mucosal samples from healthy individuals and 33 HNSCC tumor samples for seven genes selected. The GNG7 gene showed no methylation in normal mucosal samples $(0 / 14)$ and normal salivary rinses $(0 / 17)$. The methylation rate was $61 \%$ (20/33) on the promoter region of the GNG7 gene in primary HNSCC tumor samples and these tumors harbored high methylation values, mostly between 10 and $100 \%$. TXNIP, TUSC2, PPFIBP2, GALNT10 and MAP2K3 demonstrated varying degrees of methylation on their promoter regions in normal mucosa, normal salivary rinses and HNSCC tumor samples respectively (Fig. 4). We observed no methylation in the promoter region of MAP3K3 [0/14 (0), 0/18 (0) and $0 / 33(0)]$ gene in normal mucosa, normal salivary rinses and HNSCC tumor samples, respectively.

\section{Discussion}

In the present study, we combined a proven pharmacologically demethylating-unmasking strategy with an expanded $47 \mathrm{~K}$ expression microarray platform to identify novel cancerspecific methylated genes. Among the 47,000 transcripts of the Affymetrix Human Genome U133 Plus 2.0 expression arrays, we first identified seven genes of interest. We then performed QMSP on 22 saliva and 14 mucosal samples from healthy individuals and $33 \mathrm{HNSCC}$ tumor samples for the seven genes selected and verified 3 genes showing hypermethylation in the promoter regions of HNSCC tissues and minimal or absent methylation in normal salivary rinses and mucosal samples. The GNG7 gene was the most marked, showing no methylation in normal mucosal samples and normal salivary rinses respectively, while $61 \%$ of primary HNSCC tumor samples were methylated. TXNIP methylation values were less than $1 \%$ in normal salivary rinses and the promoter was not methylated in normal mucosal samples, whereas the methylation levels were over $10 \%$ in tumors. Similarly, the TUSC 2 gene was found to be methylated in $0.1 \%$ of normal mucosa samples and showed almost no methylation (under $1 \%$ ) in normal salivary rinses. Other genes were not found to be useful as potential biomarkers.

We found that the guanine nucleotide-binding protein, $\gamma-7$ (GNG7) promoter was specifically methylated in HNSCC. GNG7 is located on chromosome 19 and is a member of the guanine nucleotide-binding proteins ( $G$ proteins) which are involved as a modulator or transducer in various transmembrane signaling systems. The $\beta$ and $\gamma$ chains are required for the GTPase activity, for replacement of GDP by GTP and for $G$ protein-effector interaction; they are important in the regulation of adenylyl cyclase signaling in certain regions of the brain and have a role in the formation or stabilization of a $G$ protein heterotrimer [G(olf) subunit $\alpha-\beta-\gamma-7]$ that is required for adenylyl cyclase activity in the striatum. GNG7 showed homozygous deletions in cell lines of classical
Hodgkins lymphoma (17). Decreased expression of GNG7 identified by Ray et al (18) was confirmed in pancreatic malignancies (19) and esophageal cancer (20). Expression of G- $\gamma-7$ mRNA was downregulated in extrahepatic cholangiocarcinoma (EHCC) tissue compared to pericancerous bile duct and normal bile duct tissues and in poorly differentiated EHCC tissues (21).

Thioredoxin-interacting protein is encoded by the TXNIP gene and interacts with thioredoxin and ZBTB32. This gene functions as an oxidative stress mediator by inhibiting thioredoxin activity or by limiting its bioavailability and acts as a transcriptional repressor, between transcription factors and co-repressor complexes and its overexpression induces G0/G1 cell cycle arrest and is necessary for the maturation of natural killer cells (22-25).

Tumor suppressor candidate 2 is encoded by the TUSC2 gene which is a highly conserved lung cancer candidate gene $(26,27)$. In malignant pleural mesothelioma (28) and nasopharyngeal carcinoma (29), expression of the TUSC2 gene was found to be downregulated. TUSC 2 is often deleted in lung, breast, head and neck, renal and other types of cancer (30) and was reported to be methylated in human lung cancer cells (31). Furthermore, large-scale analysis of TUSC2 expression in lung cancer and in bronchial squamous metaplastic and dysplastic lesions showed reduced expression levels of TUSC2 compared to normal hyperplastic epithelia, indicating it could be an early event in cancer progression (32).

It is known that the cell culture may influence DNA methylation and present larger stretches of methylation events and some drawbacks when compared to primary tumors (33). Therefore, as a future step, this issue may be investigated by use of a larger panel of HNSCC cell lines. However, this fact does not invalidate our findings and the data of our study showing that the GNG7 gene is a promising candidate tumor suppressor gene and biomarker for HNSCC.

It would be helpful to test independent cohorts to evaluate the utility of these genes as HNSCC biomarkers. Additional studies in larger, prospective cohorts would also determine the prognostic significance of detection of these markers in saliva, serum or plasma samples from HNSCC patients.

\section{Acknowledgements}

This study was supported by the National Institute of Dental and Craniofacial Research (R37DE012588), The National Institute of Dental and Craniofacial Research/The National Cancer Institute (SPORE-P50DE019032) and Scientific Research Projects Coordination Unit of Istanbul University (UDP-22027). S. Dasgupta is supported by Elsa U Pardee Foundation. This report/analysis is based on a web database application provided by Research Information Technology Systems (RITS)-https://www.rits.onc.jhmi.edu/.

\section{References}

1. Jemal A, Siegel R, Ward E, Murray T, Xu J, Smigal C and Thun MJ: Cancer statistics, 2006. CA Cancer J Clin 56: 106-130, 2006.

2. Fearon ER and Vogelstein B: A genetic model of colorectal tumorigenesis. Cell 61: 759-767, 1990.

3. Baylin SB, Herman JG, Graff JR, Vertino PM and Issa JP: Alterations in DNA methylation: a fundamental aspect of neoplasia. Adv Cancer Res 72: 141-196, 1998. 
4. Dulaimi E, Hillinck J, Ibanez de Caceres I, Al-Saleem T and Cairns P: Tumor suppressor gene promoter hypermethylation in serum of breast cancer patients. Clin Cancer Res 10: 6189-6193, 2004.

5. Leonhardt $\mathrm{H}$ and Cardoso MC: DNA methylation, nuclear structure, gene expression and cancer. J Cell Biochem 35 (Suppl): S78-S83, 2000.

6. Herman JG and Baylin SB: Gene silencing in cancer in association with promoter hypermethylation. N Engl J Med 349: 2042-2054, 2003.

7. Yamashita K, Upadhyay S, Osada M, et al: Pharmacologic unmasking of epigenetically silenced tumor suppressor genes in esophageal squamous cell carcinoma. Cancer Cell 2: 485-495, 2002.

8. Bernard PS and Wittwer CT: Real-time PCR technology for cancer diagnostics. Clin Chem 48: 1178-1185, 2002.

9. Eads CA, Danenberg KD, Kawakami K, Saltz LB, Blake C and Shibata D: MethyLight: a high-throughput assay to measure DNA methylation. Nucleic Acids Res 28: E32, 2000.

10. Cottrell SE and Laird PW: Sensitive detection of DNA methylation. Ann NY Acad Sci 983: 120-130, 2003.

11. Jerónimo C, Usadel H, Henrique R, Oliveira J, Lopes C, Nelson WG and Sidransky D: Quantitation of GSTP methylation in non-neoplastic prostatic tissue and organ-confined prostate adenocarcinoma. J Natl Cancer Inst 93: 1747-1752, 2001.

12. Tokumaru Y, Yamashita K, Osada M, et al: Inverse correlation between cyclin A1 hypermethylation and p53 mutation in head and neck cancer identified by reversal of epigenetic silencing. Cancer Res 64: 5982-5987, 2004.

13. Li C and Wong WH: Model-based analysis of oligonucleotide arrays: Expression index computation and outlier detection. Proc Natl Acad Sci USA 98: 31-36, 2001

14. Tusher VG, Tibshirani R and Chu G: Significance analysis of microarrays applied to the ionizing radiation response. Proc Natl Acad Sci USA 98: 5116-5121, 2001.

15. Carvalho AL, Jeronimo C,Kim MM,et al: Evaluation of promoter hypermethylation detection in body fluids as a screening/ diagnosis tool for head and neck squamous cell carcinoma. Clin Cancer Res 14: 97-107, 2008

16. Li LC and Dahiya R: MethPrimer: designing primers for methylation PCRs. Bioinformatics 18: 1427-1431, 2002.

17. Giefing M, Arnemann J, Martin-Subero JI, et al: Identification of candidate tumour suppressor gene loci for Hodgkin and ReedSternberg cells by characterisation of homozygous deletions in classical Hodgkin lymphoma cell lines. Br J Haematol 142: 916-924, 2008.

18. Ray K, Kunsch C, Bonner LM and Robishaw JD: Isolation of cDNA clones encoding eight different human $\mathrm{G}$ protein gamma subunits, including three novel forms designated the gamma-4, gamma-10, and gamma-11 subunits. J Biol Chem 270: 21765-21771, 1995.

19. Shibata K, Mori M, Tanaka S, Kitano S and Akiyoshi T: Identification and cloning of human G-protein gamma 7, downregulated in pancreatic cancer. Biochem Biophys Res Commun 246: 205-209, 1998.
20. Ohta M, Mimori K, Fukuyoshi Y, et al: Clinical significance of the reduced expression of $G$ protein gamma 7 (GNG7) in oesophageal cancer. Br J Cancer 98: 410-417, 2008.

21. Wang M, Gong B, Li Y and Wang Y: Human G-protein gamma 7 in extrahepatic cholangiocarcinoma and its clinicopathological significance. Hematol Oncol Stem Cell Ther 3: 66-70, 2010.

22. Wollman EE, d'Auriol L, Rimsky L, et al: Cloning and expression of a cDNA for human thioredoxin. J Biol Chem 263: 15506-15512, 1988.

23. Meng L, Wong JH, Feldman LJ, Lemaux PG and Buchanan BB: A membrane-associated thioredoxin required for plant growth moves from cell to cell, suggestive of a role in intercellular communication. Proc Natl Acad Sci USA 107: 3900-3905, 2010.

24. Nishiyama A, Matsui M, Iwata S, et al: Identification of thioredoxin-binding protein-2/vitamin $\mathrm{D}(3)$ up-regulated protein 1 as a negative regulator of thioredoxin function and expression. J Biol Chem 274: 21645-21650, 1999.

25. Han SH, Jeon JH, Ju HR, et al: VDUP1 upregulated by TGF-beta1 and 1,25-dihydroxyvitamin $\mathrm{D}_{3}$ inhibits tumor cell growth by blocking cell-cycle progression. Oncogene 22: 4035-4046, 2003

26. Fischer WH and Schubert D: Characterization of a novel plateletderived growth factor-associated protein. J Neurochem 66 : 2213-2216, 1996.

27. Kondo M, Ji L, Kamibayashi C, et al: Overexpression of candidate tumor suppressor gene FUS1 isolated from the 3p21.3 homozygous deletion region leads to G1 arrest and growth inhibition of lung cancer cells. Oncogene 20: 6258-6262, 2001.

28. Ivanova AV, Ivanov SV, Prudkin L, et al: Mechanisms of FUS1/ TUSC2 deficiency in mesothelioma and its tumorigenic transcriptional effects. Mol Cancer 8: 91, 2009.

29. Zhou YB, Huang ZX, Ren CP, Zhu B and Yao KT: Screening and preliminary analysis of the apoptosis- and proliferation-related genes in nasopharyngeal carcinoma. Nan Fang Yi Ke Da Xue Xue Bao 29: 645-647, 2009 (In Chinese).

30. Lerman MI and Minna JD: The 630-kb lung cancer homozygous deletion region on human chromosome 3 p21.3: identification and evaluation of the resident candidate tumor suppressor genes. The international lung cancer chromosome $3 \mathrm{p} 21.3$ tumor suppressor gene consortium. Cancer Res 60: 6116-6133, 2000.

31. Uno F, Sasaki J, Nishizaki M, et al: Myristoylation of the fus1 protein is required for tumor suppression in human lung cancer cells. Cancer Res 64: 2969-2976, 2004.

32. Prudkin L, Behrens C, Liu DD, et al: Loss and reduction of FUS protein expression is a frequent phenomenon in the pathogenesis of lung cancer. Clin Cancer Res 14: 41-47, 2008.

33. Hennessey PT, Ochs MF, Mydlarz WW, Hsueh W, Cope L, Yu W and Califano JA: Promoter methylation in head and neck squamous cell carcinoma cell lines is significantly different than methylation in primary tumors and xenografts. PLoS One 6: e20584, 2011. 